\title{
Obstructive Sleep Apnea Treatment with a New Oral Appliance: Somnodent ${ }^{\circledR}$
}

\author{
Domenico Ciavarella $^{1}$, Antonio Sabatucci ${ }^{2}$, Mario Mastrovincenzo ${ }^{2}$, Vincenzo Parziale ${ }^{3}$, \\ Roberto Sabato ${ }^{4}$, Maria Pia Foschino Barbaro ${ }^{4}$, Nicola Giannone ${ }^{1}$, Fabio Salcuni ${ }^{1}$, \\ Maurizio Bossù ${ }^{5}$ and Lorenzo Lo Muzio ${ }^{*}, 1$
}

\author{
${ }^{I}$ Department of Surgical Sciences, Faculty of Medicine, School of Dentistry, University of Foggia, Italy \\ ${ }^{2}$ Department of Oral Sciences, Faculty of Medicine, School of Dentistry, Politecnic University of Marche, Italy \\ ${ }^{3}$ Department of Surgical Sciences, Faculty of Medicine, School of Dentistry, University of L' Aquila, Italy \\ ${ }^{4}$ Institute of Respiratory Disease, Department of Medical and Occupational Sciences, University of Foggia, Italy \\ ${ }^{5}$ Department of Dentistry, University of Rome, La Sapienza, Italy
}

\begin{abstract}
Obstructive sleep apnea (OSA) is a disorder in which recurrent closure of the upper airways occurs during sleep. Patients habits correction and the treatment of hypothyroidism, if present, may help to resolve this condition, otherwise other treatements may be tried: nasal continuous positive pressure (nCPAP), surgical treatment, or the application of oral appliances. In this paper a case control study with a new type of mandible-advancement device (MAD) $\left(\right.$ Somnodent ${ }^{\circledR}$ ) used in OSA treatment is presented.

Materials and Methods: 17 patients affected by OSA with a mean age of 53.52 years (range 32 - 63) had been evaluated. Cephalometric assessment of head radiographs taken in the lateral plane was performed; mandible plane-hyoid (MP-H) distance and the pharyngeal anterior space distance (PAS) were measured. Body mass index (BMI) was calculated, and mean oxygen saturation $\left(\mathrm{SaO}_{2}\right)$ and the respiratory disturbance index (AHI) was evaluated. Each patient received a complete overnight polysomnography.

Results: AHI reduction with MAD was considered extremely significant $(\mathrm{P}<0.0001)$. The reduction of apnea/hypopnea index induced significant variation of $\mathrm{SaO}_{2}(86.859 \%$ vs $93.906 \%$, without and with MAD, respectively: $\mathrm{p}<0.0001)$.

Conclusions: OSA therapy with MAD seems very effective and, compared to other treatments (in particular nCPAP), may enhance patient's compliance.
\end{abstract}

Keywords: Obstructive sleep apnea, somnodent, oral appliance.

\section{INTRODUCTION}

Obstructive sleep apnea (OSA) is a disorder in which recurrent closure of the upper airways occur during sleep. These frequent arousals are the primary cause of excessive daytime somnolence [1], associated with impaired daytime cognitive function, and are recognized as a cofactor in the etiology of road traffic accidents. Sleep apnea may also be associated with increased cardiovascular and increased cerebrovascular morbidity and mortality $[2,3]$. Many techniques have been used to evaluate the upper airways in patients with obstructive sleep apnea. These techniques have included cephalometry, computed tomography, magnetic resonance imaging, fluoroscopy, acoustic reflection studies and polysomnography (PSG) [4-6]. Electroencephalogram, eye movements, postural muscle tone, oxygen saturation $\left(\mathrm{SaO}_{2}\right)$, air flow, respiratory effort and heart rate are measured with a complete PSG. Full PSG evaluates the

*Address correspondence to this author at the Department of Surgical Sciences, Faculty of Medicine, School of Dentistry, University of Foggia, Viale Pinto, 1, 71100 Foggia, Italy; Tel: +39 0881 588041; Fax: +39 0881 588041; E-mail: 1lomuzio@tin.it apnea index (AI) and respiratory disturbance index (AHI). AI indicates the number of apneas per hour. AHI indicates the number of apneas/hypopneas per hour of sleeping (this is also called AHI apnea/hypopnea index).

An estimated $82 \%$ of men and $92 \%$ of women with moderate to severe sleep apnea remain undiagnosed [7].

OSA treatment is chosen, based on patients clinical features. Its resolution may occurr by losing weight, if the patient is obese, avoid sleeping in the supine position, if the OSA is position dependent, avoid alcohol consumption during the evening and treat hypothyroidism, if present. When these approaches are proved to be unsuccessful, other treatments should be choosen: nasal continuous positive pressure (nCPAP), surgical treatment or the application of oral appliances.

Actually, continuous or bi-level positive airway pressure (CPAP/biPap) represents the gold standard in the treatment of obstructive sleep-related breathing disorders [8]. Many authors suggest that appropriate use of nCPAP in the patients with OSA may be required to decrease implications for cardiovascular morbidity and mortality [9]. 
Patients with obstructive sleep apnea and high risk for morbidity may be treated with surgical management with change of upper airways obstruction. Surgical treatment of obstructive sleep apnea is limited to about $15 \%$ of the patients and, usually, reserved for those in whom a trial of nasal CPAP fails. In these patients, surgery is the elective form of therapy. Brigance et al. showed how AHI in patients treated with surgical management of OSA significantly improved [10].

CPAP and surgery treatment reqiure a great patients collaboration. Many authors did not treat severe OSA with mandible-advancement devices (MAD) [11, 12]. In this paper a case control study with a new type of MAD (Somnodent ${ }^{\circledR}$ ) used in the patients with differing severities of OSA is presented.

\section{MATERIALS AND METHODS}

17 patients affected by OSA with a mean age of 53.52 years (range 32 - 63) had been evaluated. The absence of temporo-mandibular joint disease and the presence of healthy periodontal ligament were additional inclusion criteria. On each patient head radiographs, with and without oral appliance, were taken in the lateral plane with the head fixed in a cephalostat with a film-focus distance of $4 \mathrm{~m}$ and a midsagittal-to-film distance of $0.1 \mathrm{~m}$. Cephalometric assessment of radiographs was performed. The cephalometric protocol measurements and landmarks have been described by Lowe et al. [13, 14]. The maxillary anatomical landmarks were: Anterior Nasal Spine (ANS), maxilla point A (deepest midline point on the maxillary alveolus between ANS and the maxillary alveolar crest), posterior nasal spine (PNS), and the tip of the posterior spine of the palatine bone in the hard palate. The cranium landmarks were: Nasion $(\mathrm{N})$, the anterior point of the frontonasal suture; Sella $(\mathrm{S})$, the center of the sella turcica. The Mandible landmarks were: Gonion (Go), the most lateral external point at the junction of the horizontal and ascending ramus of the mandible; Menton (Me), the lowest point on the body outline of the mandible symphysis. The hyoid landmarks were: Hyoidale $(\mathrm{H})$, the most superioranterior point on the hyoid bone. On the lateral radiograph anterior pharynx space (PAS), Hyoid-Mandible plane distance (MP-H) and ANB were measured. PAS (mm) is the distance between the posterior pharyngeal wall and the dorsal surface of the base of the tongue, measured on the line that intersects $\mathrm{GO}$ and $\mathrm{B}$ point. The distance between $\mathrm{H}$ to Mandible Plane (the joining Me and Go) was measured too. To determine the skeletal class, the ANB-angle was individualized.

Body mass index (BMI) was calculated, mean oxygen saturation $\left(\mathrm{SaO}_{2}\right)$ and the respiratory disturbance index $\mathrm{AHI}$ were evaluated. Each patient received a complete overnight polysomnography (PSG). All subjects were evaluated for one night in a Sleep Laboratory using a portable device, the Embletta system (Flaga, Reykjavik, Iceland). Recording was performed after one night of adaptation to the hospital setting. Airflow was monitored by a nasal cannula and by oral thermistore. The thoracic-abdominal movements of all subjects were detected through two piezoelectric belts.
Overnight continuous recordings of oxygen saturation were obtained by finger pulse oximetry. Snoring was recorded by a microphone placed at the neck, and note was taken of ECG findings and sleep position. Apnea was defined as the cessation of airflow lasting $10 \mathrm{sec}$; and hypopnea was defined as a discrete reduction (two thirds) of airflow and/or abdominal rib-cage movements lasting $10 \mathrm{sec}$ associated with a $>3 \%$ decrease in oxygen saturation. The number of events per hour was obtained by dividing the total number of events by the total sleep time (TST) and was defined as the apnea/hypopnea index (AHI). Also the oxygen desaturation index (ODI) was measured, as the number of arterial oxyhemoglobin saturation dips $>=3 \%$. Nocturnal hypoxemia was evaluated in terms of percentage of total sleep time with oxyhemoglobin saturation $<90 \%$. Sleep apnea was defined using AHI $>=10$ per hour. On each patient the test was repeated with oral appliance three months later.

For each patient dental impressions and an interocclusal wax in protrusive comfortable mandible position were used to fabricate the MAD (Fig. 1). Mean mandible advancement was $4,73 \mathrm{~mm}$ (range, 1,5 to $10 \mathrm{~mm}$ ). The design of this type of MAD was in two acrylic splints. The mandible splint had two buccal flanges angled at $70^{\circ}$, which fitted against buccal blocks on the upper splint to prevent the posterior movement of the mandible. For its retention, tooth undercuts and Adams hooks were used. Flanges length was regulated on each patient to obtain a comfortable wearing.

\section{RESULTS}

Mean MP-H was $21.67 \mathrm{~mm}$ (range 10 to $30 \mathrm{~mm}$ ) and mean PAS was $6.85 \mathrm{~mm}$ (range 4 to $9 \mathrm{~mm}$ ). With the above mentioned mandible advancement, we have very relevant results and improvement as regards AHI and oxygen saturation, whose data with and without MAD application are detailed in Table 1. MAD application caused a very significant AHI reduction; in fact, mean AHI without MAD was 31.659, while it was reduced to 8.735 with MAD application $(\mathrm{P}<0.0001)$.

The reduction of apnea/hypopnea index induced significant variation of oxygen saturation $\left(\mathrm{SaO}_{2}\right)$ as showed by measurements made during polysomnography test (86.859\% vs $93.906 \%$, without and with MAD, respectively: $\mathrm{p}<0.0001)$.

\section{DISCUSSION}

OSA is recognized to be a very common clinical condition that affects an estimated $4-5 \%$ of middle aged man and $1-2 \%$ of middle aged woman [15].

The definitions of apnea, hypopnea, sleep disordered breathing, and/or OSA are discussed in detail in many researches [16-18].

Pathogenesis of OSA is still unclear. Clinically two forms of OSA are decrypted: the primary and the secondary form (Fig. 2). An alteration of the central nervous system is present in the primary form (central form) in which alteration of ventilation is related to an incoming of the nervous stimulus at the respiratory system. This type of OSA has a more complex diagnosis and is more difficult to treat than the secondary form. 

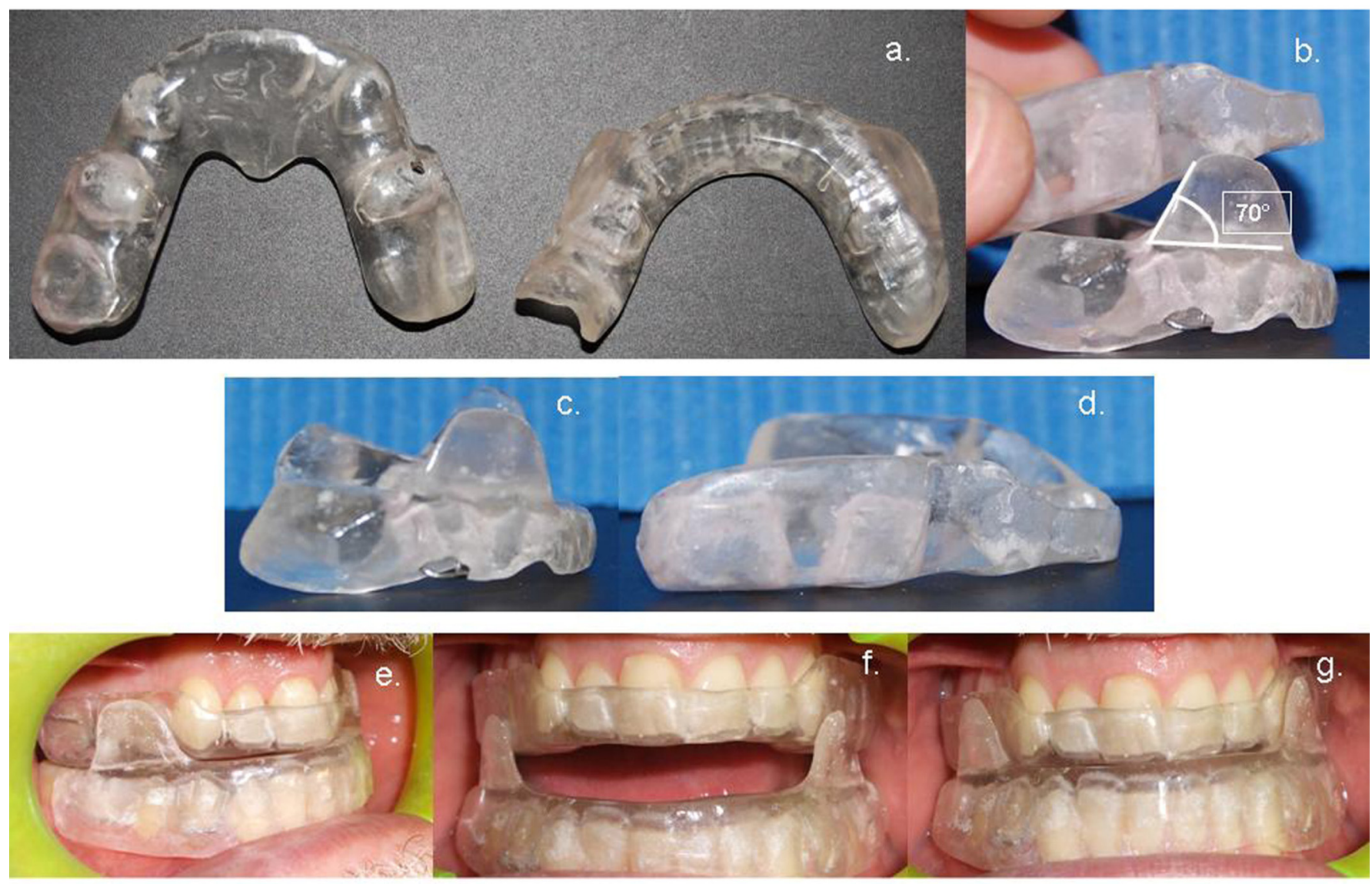

Fig. (1). (a) Somnodent ${ }^{\circledR}$; (b) acrylic splints with buccal flanges angled at $70^{\circ}$; (c) lower splint; (d) upper splint; (e) patients with MAD lateral vision; (f) patients with MAD in resting position; (g) patients with MAD in close mouth.

Table 1. Patients Data with and without Mandible-Advancement Device Application

\begin{tabular}{|c|c|c|c|c|c|c|c|c|}
\hline & & Mean & SEM & Median & Min & Max & 95\% CI & $p$-Value (Paired t Test) \\
\hline \hline $\begin{array}{c}\text { AHI } \\
\text { (Apnea/Hypopnea } \\
\text { Index) }\end{array}$ & without MAD & 31.659 & 4.151 & 27.700 & 11.500 & 79.000 & $22.8-40.46$ & $<0.0001$ \\
\cline { 2 - 9 } & with MAD & 8.735 & 2.021 & 6.700 & 0.4000 & 29.000 & $4.45-13.02$ & $85.7-87.9$ \\
\hline $\begin{array}{c}\text { Mean Oxygen } \\
\text { Saturation (\%) }\end{array}$ & without MAD & 86.859 & 0.5046 & 87.000 & 84.000 & 91.000 & $<0.0001$ \\
\cline { 2 - 9 } & with MAD & 93.906 & 0.2787 & 94.000 & 92.000 & 96.000 & $93.3-94.4$ & \\
\hline
\end{tabular}

SEM: Standard error of means.

CI: Confidence Interval.

Significance level: $\mathrm{p}<0.05$

Reduction of upper airways width, obesity [16], obstructive factors such as adenoide-tonsillae hypertrophy, PAS reduction, redundant soft palate and prominent base of tongue were included in the secondary form classification [17].

Airways collapse may occur at the level of the soft palate, at the base of the tongue against the posterior wall of the pharynx, or at both locations [19].

Typically, after at least 10 seconds without adequate ventilation the patient wakens briefly, inhales, and then returns to sleep.

This cycle repeats itself throughout the night, in some cases occurring hundreds of times. Arousals may be due to apnea (complete cessation of breathing for more than 10 seconds), hypopnea (a $50 \%$ reduction in thoracoabdominal movement accompanied by a decrease of $4 \%$ or more in oxygen saturation), or both. Oxygen levels can be depressed to alarming levels when apneas continue for long periods [20-22].

In recent years, great interest received the MAD. In fact, the anterior mandible repositioning causes complex changes within the lateral pharyngeal walls, tongue, soft palate, epiglottis and genioglossus muscle that induce an improvement of respiratory dynamics [23].

MAD effects in cranium lateral radiography has been evaluated. The PAS increase indicated the intentional effect of MAD (Fig. 3). 


\section{Sleep Apnea}

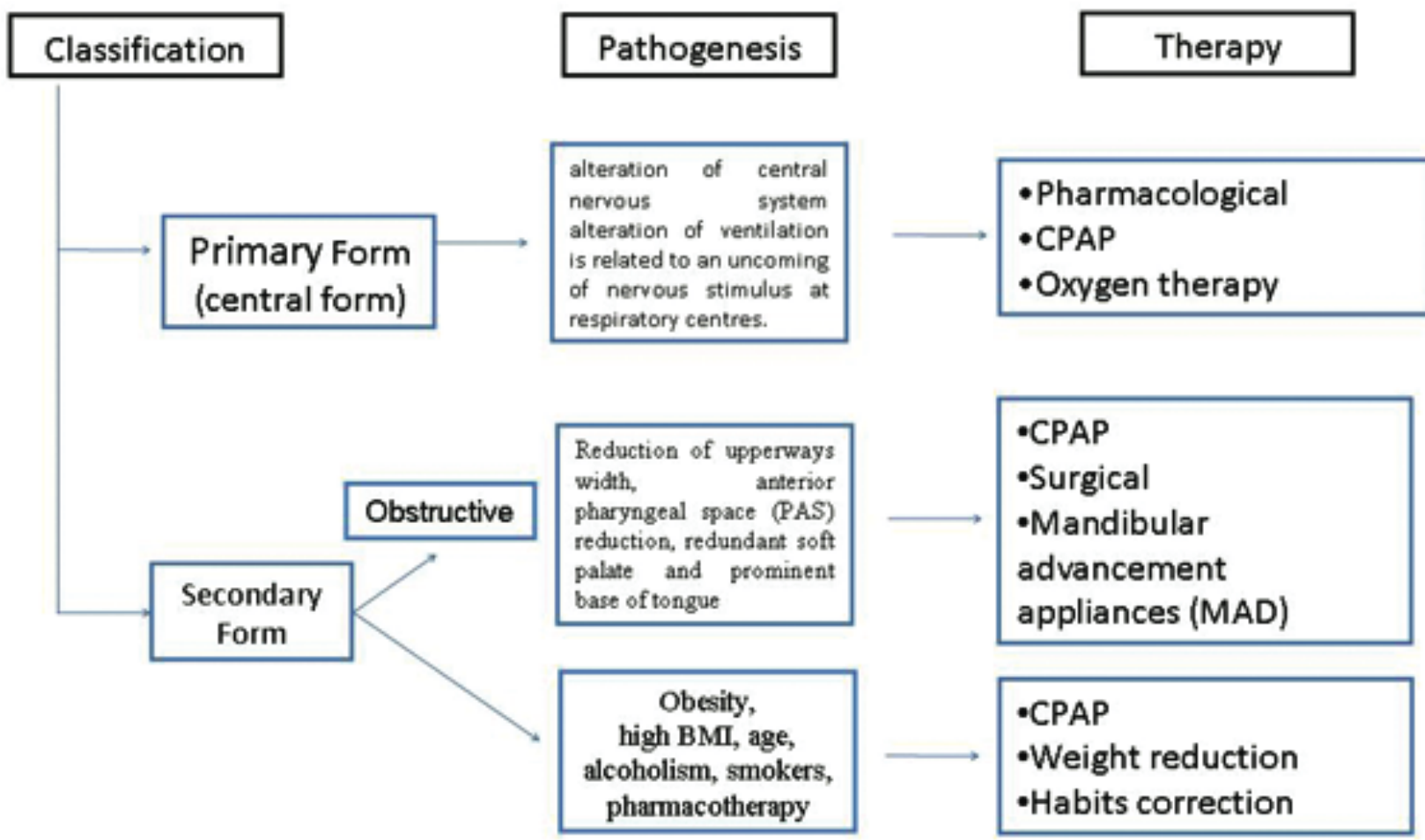

Fig. (2). Classification, pathogenesis and therapy of OSAs.
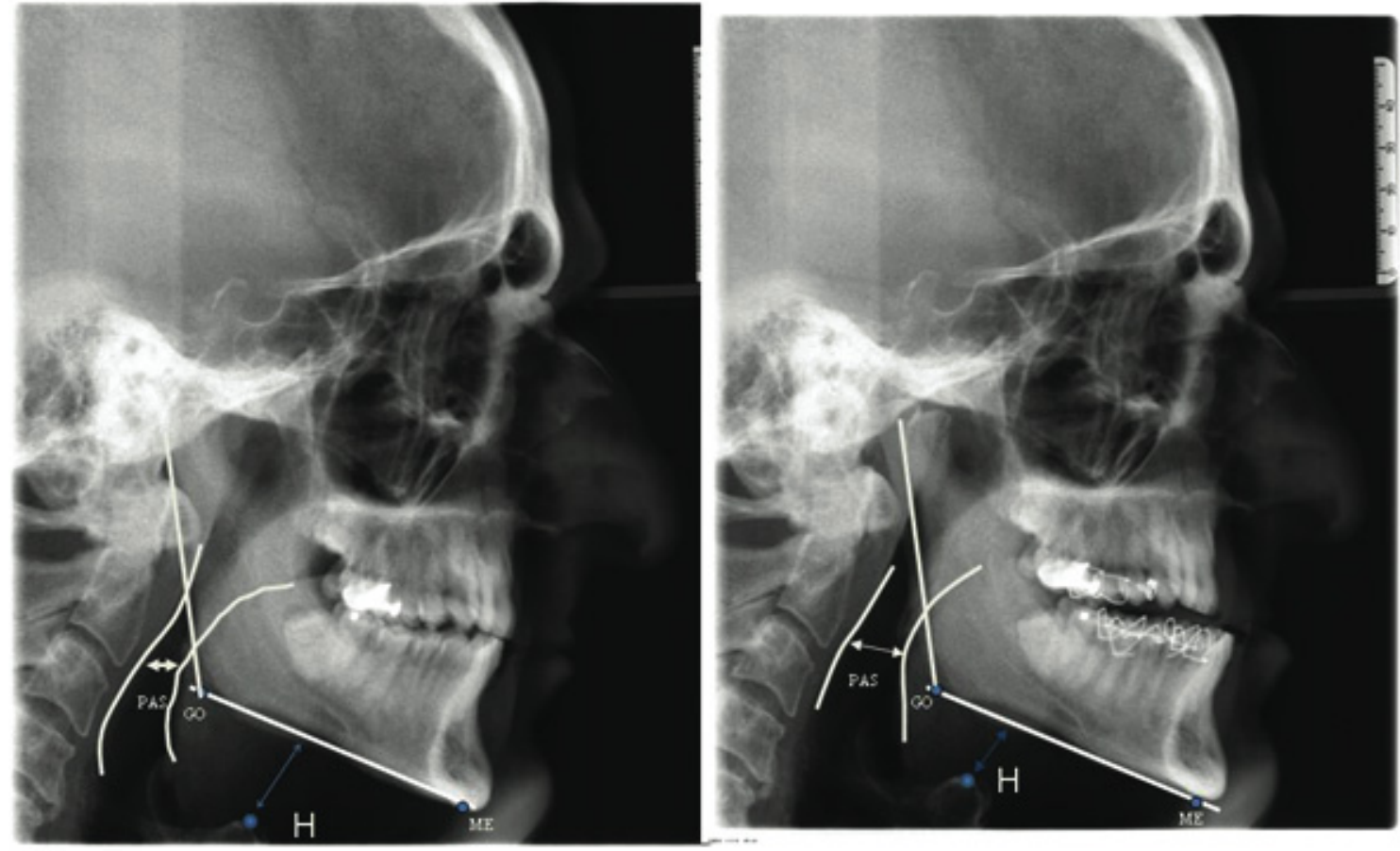

Fig. (3). Radiography of a patient. Note mandible advancement with MAD, the effect on PAS and hyoid bone position modification.

This type of therapy is better for a direct low cost to patients and health maintenance organizations.

The properties of the oral appliance has been showed by Ekchart [24], who reviewed the state of the art of oral appliances and laid out the properties of the devices to be considered, ranging from their reliability at stopping, to the permitted degree of freedom in lateral movements.

To reduce the complications that are commonly associated with MAD, it is very important to establish correct criteria for patients selection. The literature suggests 
two types of complications: minor (i.e. tooth pain, excess salivation, dry mouth, TMJ discomfort and muscle pain) and severe complications (i.e. TMJ dysfunction, gagging, tooth movement, and intractable muscle pain). MAD [25] may not be used regularly due to complications.

The American sleep disorders association suggest to use MAD only in the patients with no severe OSAs and whenever CPAP failed or it is refused by patients [25].

A great and significant $(\mathrm{P}<0.0001)$ AHI reduction by using MAD has been showed in the present study. This had a beneficial effect also in mean oxygen saturation measured by a complex poliysomnogram; in fact, during sleep with MAD $\mathrm{SaO}_{2}$ was $93.906 \%$ vs $86.859 \%$ without MAD, and the difference was statistically significant. The $\mathrm{SaO}_{2}$ increase may have important implications in the possible reduction of high blood pressure related to secondary policitemy.

\section{CONCLUSIONS}

A new OSA therapy with MAD may enhance patients compliance.

The use of MAD in OSAs seems very effective, Thus, capable to reduce complications associated to this condition. In addition, while CPAP is actually the gold standard therapy for OSA, due to its complexity patients are often reluctant to use it; conversely, MAD has the advantage to be comfortable and very easy to use, thus, very good patients compliance can be obtained quite simply. For these reasons MAD seems very promising and worth of further research in OSA therapy.

\section{REFERENCES}

[1] Johns F, Strollo P, Bukley M, Costantino J. The influence of craniofacial structure on obstructive sleep apnea in young adults. J Oral Maxillofac Surg 1998; 56: 596-602.

[2] Koskenvuo M, Kaprio J, Telakivi T, Partinen M, Heikkila K, Sarna $\mathrm{S}$. Snoring is a risk factor for ischemic heart disease and stroke in men. Br Med J 1987; 294: 16-9.

[3] Partinen M, Jamieson A, Guilleminault C. Long term outcome from obstructive sleep apnea patients. Chest 1989; 94: 1200-4.

[4] Acar B, Karabulut H, Babademez MA, Ciftci B, Karasen RM. Evaluation of submucosal minimally invasive lingual excision technique for treatment of obstructive sleep apnea/hypopnea syndrome. Otolaryngol Head Neck Surg 2009; 140: 281; author reply

[5] Shepard J, Geller W, Guilleminault C. Evaluation of the upper airway in patients with obstructive sleep apnea. Sleep 1991; 14: 36.

[6] Fleetham JA, Ferguson KA, Lowe AA, Ryan CF. Oral appliance therapy for the treatment of obstructive sleep apnea. Sleep 1996; 19: S288-90.
[7] Chung F, Elsaid H. Screening for obstructive sleep apnea before surgery: why is it important? Curr Opin Anaesthesiol 2009; 22: 405-11.

[8] Martinez-Garcia MA, Soler-Cataluna JJ, Ejarque-Martinez L, et al. CPAP treatment reduces mortality in ischemic stroke patients with obstructive sleep apnea. Am J Respir Crit Care Med 2009; 180(1): 36-41.

[9] Ishida K, Kato M, Kato $\mathrm{Y}$, et al. Appropriate use of nasal continuous positive airway pressure decreases elevated C-reactive protein in patients with obstructive sleep apnea. Chest 2009; 13(1): $125-9$.

[10] Brigance JS, Miyamoto RC, Schilt P, et al. Surgical management of obstructive sleep apnea in infants and young toddlers. Otolaryngol Head Neck Surg 2009; 140: 912-6.

[11] Randerath WJ, Heise M, Hinz R, Ruehle KH. An individually adjustable oral appliance $v s$ continuous positive airway pressure in mild-to-moderate obstructive sleep apnea syndrome. Chest 2002; 122: 569-75.

[12] Ferguson KA. The role of oral appliance therapy in the treatment of obstructive sleep apnea. Clin Chest Med 2003; 24: 355-64.

[13] Lowe AA, Ono T, Ferguson KA, Pae EK, Ryan CF, Fleetham JA. Cephalometric comparisons of craniofacial and upper airway structure by skeletal subtype and gender in patients with obstructive sleep apnea. Am J Orthod Dentofacial Orthop 1996; 110: 653-64.

[14] Lowe AA, Santamaria JD, Fleetham JA, Price C. Facial morphology and obstructive sleep apnea. Am J Orthod Dentofacial Orthop 1986; 90: 484-91.

[15] Young T, Palta M, Dempsey J. The occurence of sleep disordered breathing among middle aged adults. N Engl J Med 1993; 328: 1230-5.

[16] Antczak J, Horn B, Richter A, Jernajczyk W, Bodenschatz R, Schimdt WEW. The influence of obesity on sleep quality in male sleep apnea patients before and during therapy. J Physiol Pharmacol 2008; 59(Suppl 6): 123-34.

[17] Don DM, Geller KA, Koempel JA, Ward SD. Age specific differences in pediatric obstructive sleep apnea. Int J Pediatr Otorhinolaryngol 2009; 73: 1025-8.

[18] George CFP. Diagnostic techniques in obstructive sleep apnea. Prog Cardiovasc Dis 1999; 41: 355-66.

[19] Wilms D, Popovich J, Conway W, Fujita S, Zorick F. Anatomic abnormalities in obstructive sleep apnea. Ann Otol Rhinol Laryngol 1982; 91: 595-6.

[20] Cohen R. Obstructive sleep apnea. Oral Surg Oral Med Oral Pathol Oral Radiol Endod 1998; 85: 388-92.

[21] Cohen R. Obstructive sleep apnea: oral appliance therapy and severity of condition. Oral Surg Oral Med Oral Pathol Oral Radiol Endod 1998; 85: 388-92.

[22] Pack A. Obstructive sleep apnea. Adv Intern Med 1994; 39: $517-$ 67.

[23] Moore EK. Oral appliance treatment for obstructive sleep apnea. Oper Tech Otolaryngol 2007; 18: 52-6.

[24] Eckhart J. Comparison of oral devices for snoring. CDA J 1998; 26: 611-23.

[25] American S, Asociations prectice parameters for the treatment of snoring and obstructive sleep apnea with oral appliances. Sleep 1995; 18: 511-3. 